\title{
MicroRNA-181b is downregulated in non-small cell lung cancer and inhibits cell motility by directly targeting HMGB1
}

\author{
YUN LIU $^{1,2}, \mathrm{XU} \mathrm{HU}^{1,2}$, DAOKUI XIA ${ }^{1,2}$ and SONGLIN ZHANG ${ }^{1,2}$ \\ ${ }^{1}$ Department of Cardiothoracic Surgery, The First College of Clinical Medical Science, China Three Gorges University; \\ ${ }^{2}$ Department of Cardiothoracic Surgery, Yichang Central People's Hospital, Yichang, Hubei 443000, P.R. China
}

Received May 19, 2015; Accepted August 19, 2016

DOI: $10.3892 / 01.2016 .5198$

\begin{abstract}
The expression of microRNA-181b (miR-181b) has been investigated in various human cancers. However, the expression and functions of miR-181b in non-small cell lung cancer (NSCLC) are yet to be studied. In the present study, miR-181b expression in NSCLC tissues and cell lines was analyzed by quantitative polymerase chain reaction, and was shown to be recurrently downregulated. Following transfection of the H23 and H522 NSCLC cells lines with miR-181b, cell migration and cell invasion assays were performed to evaluate the effect of miR-181b overexpression on the cell motility. It was demonstrated that overexpression of miR-181b inhibited the migration and invasion of NSCLC cells. Subsequently, bioinformatics analysis, western blotting and luciferase reporter assays were conducted to investigate the mechanism underlying the miR-181b-mediated inhibition of NSCLC cell motility. It was found that miR-181b directly targeted high-mobility group box-1 (HMGB1) in NSCLC cells. These results reveal a novel therapeutic target, the miR-181b/HMGB1 axis, in NSCLC. Treatment approaches targeting this axis will be beneficial to prevent NSCLC from becoming invasive.
\end{abstract}

\section{Introduction}

Lung cancer is a life-threatening malignant tumor that is associated with the highest morbidity and mortality rates globally (1). Lung cancer can be classified into two major pathological categories: Small cell lung cancer (SCLC) and non-SCLC (NSCLC) (2). NSCLC, as the predominant form of lung cancer, accounts for nearly $85 \%$ of all lung cancer cases (3). Due to the increasing rate of cigarette smoking and air pollution, it has become a common malignancy in developing countries (4). Although the survival rate for lung

Correspondence to: $\mathrm{Dr} \mathrm{Xu} \mathrm{Hu}$, Department of Cardiothoracic Surgery, Yichang Central People's Hospital, 183 Yilin Road, Yichang, Hubei 443000, P.R. China

E-mail: yunliu99570@163.com

Key words: non-small cell lung cancer, high-mobility group box-1, microRNA-181b, metastasis cancer has increased due to improvements in diagnosis and treatment, the prognosis of NSCLC remains poor. The 5- and 10 -year survival rates remain at $<15$ and $<7 \%$, respectively (5). Metastasis is the major cause of mortality among patients with NSCLC (6). Tumor metastasis is a complex biological process, which involves cell migration, invasion, matrix degradation and angiogenesis (7). Therefore, the identification of predictive molecular factors and an understanding of the underlying molecular mechanisms during tumor metastasis are critical to further improve the survival of NSCLC patients.

Over the past few years, microRNAs (miRNAs/miRs) have been demonstrated to be involved in the pathogenesis of human lung cancer (8). miRNAs, a class of small non-coding RNAs ( $\sim 22$ nucleotides long), are widely expressed in eukaryotes and regulate the expression of their target mRNAs by base pairing with mRNAs in the 3'-untranslated region (UTR), leading to mRNA cleavage or translation repression (9-12). Thus, they are known to have essential roles in a variety of physiological and pathological processes, including the cell cycle, proliferation, migration, invasion, apoptosis, differentiation and development (13-15). Recent studies have demonstrated that miRNAs could also function as tumor suppressors or oncogenes, and that they are aberrantly expressed in numerous types of human cancer (16). Upregulated miRNAs function as oncogenes through the negative regulation of tumor suppressor genes. By contrast, downregulated miRNAs function as tumor suppressor genes and inhibit cancer through the regulation of oncogenes $(17,18)$. One miRNA can regulate a number of target genes, while one gene can also be regulated by multiple miRNAs. It has been estimated that $>60 \%$ of all human genes are regulated by miRNAs (19). Therefore, the identification of miRNA targets is essential in order to understand the function of miRNAs in cancer development and progression.

miR-181b has been reported to be frequently downregulated in various tumors (20-22). However, the expression and functions of miR-181b in NSCLC are yet to be investigated. The objective of the present study was to elucidate the expression and effect of miR-181b in NSCLC, and to investigate its underlying mechanisms.

\section{Materials and methods}

Clinical specimens and cell culture. The protocol of the present study was approved by the Protection of Human Subjects 
Committee of Yichang Central People's Hospital (Yichang, Hubei, China). Written informed consent was provided by all patients in this study. Primary cancer tissues and matched normal adjacent tissues (NATs; located $>3 \mathrm{~cm}$ from the tumor) were collected from 62 NSCLC patients, including 24 male and 38 female patients (age range, 28-74 years), who had undergone surgical resection at Yichang Central People's Hospital between January 2012 and July 2014. None of the patients had received any pre-operative cancer treatment, such as radiotherapy or chemotherapy. Tissues were rapidly snap-frozen in liquid nitrogen and stored at $-80^{\circ} \mathrm{C}$ refrigerator for subsequent experiments. The clinical data of the NSCLC patients were also collected.

The human NSCLC cell lines, H23 and H522, were purchased from the Shanghai Institute of Biochemistry and Cell Biology (Shanghai, China). The H23 and H522 cells were cultured in RPMI-1640 medium supplemented with $10 \%$ fetal bovine serum (FBS; Gibco; Thermo Fisher Scientific, Inc., Waltham, MA, USA) in a $5 \% \mathrm{CO}_{2}$ cell incubator at $37^{\circ} \mathrm{C}$.

$R N A$ isolation and reverse transcription-quantitative polymerase chain reaction ( $R T-q P C R)$. Total RNA was extracted with TRIzol reagent (Invitrogen; Thermo Fisher Scientific Inc.) according to the manufacturer's protocols. The concentration of the extracted RNA was determined using the ND-2000 spectrophotometer (NanoDrop Technologies; Thermo Fisher Scientific, Inc., Pittsburgh, PA, USA). RNA $(1 \mu \mathrm{g})$ was reverse transcribed into cDNA using a reverse transcription kit (Tiangen Biotech Co., Ltd., Beijing, China). qPCR was performed using a standard SYBR PrimeScript miRNA RT-PCR kit (Takara Bio, Inc., Otsu, Japan) on an Applied Biosystems 7500 Real-Time PCR system (Thermo Fisher Scientific, Inc., Waltham, MA, USA). U6 was used as an internal control. The primer sequences were as follows: miR-181b forward, 5'-AACATTCATTGCTGTCGGTG-3' and reverse, 5'-GCTGTCAACGATACGCTACGT-3'; and U6 forward, 5'-GCTTCGGCAGCACATATACTAAA-3' and reverse, 5'-GCTTCACGAATTTGCGTGTCAT-3'. The cycling conditions were as follows: $42^{\circ} \mathrm{C}$ for $5 \mathrm{~min} ; 95^{\circ} \mathrm{C}$ for $10 \mathrm{sec}$; and 40 cycles of $95^{\circ} \mathrm{C}$ for $5 \mathrm{sec}, 55^{\circ} \mathrm{C}$ for $30 \mathrm{sec}$ and $70^{\circ} \mathrm{C}$ for $30 \mathrm{sec}$. Each sample was analyzed in triplicate. The relative expression of miR-181b was analyzed using the $2^{-\Delta \Delta C q}$ method (23).

Cell transfection. Mature miR-181b mimic, miRNA mimic negative control (NC) and the luciferase reporter plasmid were purchased from GenePharma (Shanghai, China). The sequence of the miR-181b mimic was 5'-AACAUUCAU UGCUGUCGGUGGGU-3'. The sequence of the NC mimic was 5'-UUCUCCGAACGUGUCACGUTT-3'. Transfection with miR-181b mimic, $\mathrm{NC}$ or luciferase reporter plasmid was performed using Lipofectamine 2000 (Invitrogen; Thermo Fisher Scientific, Inc., Waltham, MA, USA) according to the manufacturer's protocols.

Cell migration and invasion assay. Migration and invasion analysis of the NSCLC cells was performed using Transwell chambers with an $8-\mu \mathrm{m}$ pore polycarbonate membrane (Costar; Corning Incorporated, Corning, NY, USA). For the migration assays, transfected cells $\left(5 \times 10^{4}\right)$ in $200 \mu$ l RPMI-1640 medium with $0.1 \%$ serum were placed into the upper chamber. For the invasion assays, transfected cells $\left(5 \times 10^{4}\right)$ in $200 \mu \mathrm{l}$ RPMI-1640 medium with $0.1 \%$ serum were placed into the upper chamber coated with Matrigel (BD Biosciences, San Jose, CA, USA). In each assay, a volume of $0.5 \mathrm{ml}$ RPMI-1640 medium with $20 \%$ FBS was then added to the lower chamber as a chemoattractant. The cells were then incubated for another $12 \mathrm{~h}$ for the migration assay and for $24 \mathrm{~h}$ for the invasion assay. The cells that did not migrate or invade through the pores were carefully wiped away with cotton wool. Next, the inserts were fixed with $100 \%$ methanol (Shanghai Macklin Biochemical Co., Ltd., Shanghai, China), stained with $0.5 \%$ crystal violet (Beyotime Institute of Biotechnology, Haimen, China) and the number of cells was counted with an inverted microscope (CKX41; Olympus, Tokyo, Japan).

Western blotting. Total cellular protein was isolated using radioimmunoprecipitation assay lysis buffer $[50 \mathrm{mM}$ Tris- $\mathrm{HCl}$ (pH 7.4), $1 \%$ NP-40, $0.25 \%$ Na-deoxycholate, $150 \mathrm{mM} \mathrm{NaCl}$, $1 \mathrm{mM}$ EDTA, $1 \mathrm{mM}$ phenylmethylsulfonyl fluoride, aprotinin, leupeptin and pepstatin $(1 \mu \mathrm{g} / \mathrm{ml}$ each), $1 \mathrm{mM} \mathrm{Na} 3 \mathrm{VO} 4$ and $1 \mathrm{mM} \mathrm{NaF}$ ) at $72 \mathrm{~h}$ post-transfection. Protein concentration was measured using a bicinchoninic acid assay kit (Nanjing KeyGen Biotech. Co., Ltd., Nanjing, China). Subsequently, equal amounts of protein were separated by $10 \%$ SDS-PAGE and electrotransferred onto polyvinylidene fluoride membranes (EMD Millipore, Billerica, MA, USA). The membranes were blocked with 5\% non-fat milk in 0.1\% TBST and incubated overnight at $4^{\circ} \mathrm{C}$ with rabbit anti-high-mobility group box-1 protein (HMGB1) monoclonal antibody (1:1,000; cat. no. ab92310; Abcam, Cambridge, UK) and mouse anti- $\beta$-actin monoclonal antibody (1:1,000; cat. no. sc-130301; Santa Cruz Biotechnology, Inc., Dallas, TX, USA). After washing with TBST, the membranes were incubated with horseradish peroxidase-conjugated goat anti-rabbit and goat anti-mouse secondary antibodies (1:5,000; cat. nos. sc-2004 and sc-2005, respectively; Santa Cruz Biotechnology, Inc.) for $1 \mathrm{~h}$ at room temperature. The protein bands were developed with enhanced chemiluminescence reagents (Pierce; Thermo Fisher Scientific, Inc., Waltham, MA, USA) and images were captured using a FluorChem imaging system (Alpha Innotech, San Leandro, CA, USA).

Luciferase assay. To determine whether HMGB1 is a direct target of miR-181b, luciferase reporter assays were performed. The luciferase reporter plasmids, pmirGLO-HMGB1-3'UTR wild-type (WT) and pmirGLO-HMGB1-3'UTR mutant (MUT), were synthesized and purified by GenePharma. The $\mathrm{H} 23$ and H522 cells were transfected with $0.5 \mu \mathrm{g}$ reporter plasmid, $40 \mathrm{nmol} \mathrm{miR-181b} \mathrm{mimic} \mathrm{or} \mathrm{NC} \mathrm{in} \mathrm{a} \mathrm{12-well}$ plate using Lipofectamine 2000 according to the manufacturer's instructions. At $48 \mathrm{~h}$ post-transfection, the activities of the firefly and renilla luciferases were determined with the Dual-Luciferase Reporter Assay System (Promega, Manheim, Germany). Each assay was replicated 3 times. The firefly luciferase activity was normalized to the renilla luciferase activity for each transfected well.

Statistical analysis. Data are presented as the mean \pm standard deviation and compared using Student's t-tests in Stata 10.0 
Table I. A comparison between miR-181b expression in non-small cell lung cancer and clinicopathological features.

\begin{tabular}{|c|c|c|c|c|}
\hline \multirow[b]{2}{*}{ Clinical features } & \multirow[b]{2}{*}{ Cases, $\mathrm{n}$} & \multicolumn{2}{|c|}{ miR-181b expression, $\mathrm{n}$} & \multirow[b]{2}{*}{ P-value } \\
\hline & & Low & High & \\
\hline Gender & & & & 0.734 \\
\hline Male & 24 & 18 & 6 & \\
\hline Female & 38 & 27 & 11 & \\
\hline Age, years & & & & 0.359 \\
\hline$<57$ & 35 & 27 & 8 & \\
\hline$\geq 57$ & 27 & 18 & 9 & \\
\hline Smoking history, years & & & & 0.407 \\
\hline$<10$ & 24 & 16 & 8 & \\
\hline$\geq 10$ & 38 & 29 & 9 & \\
\hline Tumor differentiation & & & & 0.098 \\
\hline I-II & 36 & 29 & 7 & \\
\hline III-IV & 26 & 16 & 10 & \\
\hline TNM classification & & & & 0.026 \\
\hline I & 27 & 15 & 12 & \\
\hline II & 19 & 17 & 2 & \\
\hline III+IV & 16 & 13 & 3 & \\
\hline Metastasis & & & & 0.035 \\
\hline No & 34 & 21 & 13 & \\
\hline Yes & 28 & 24 & 4 & \\
\hline
\end{tabular}

miR, microRNA; TNM, tumor-node-metastasis.

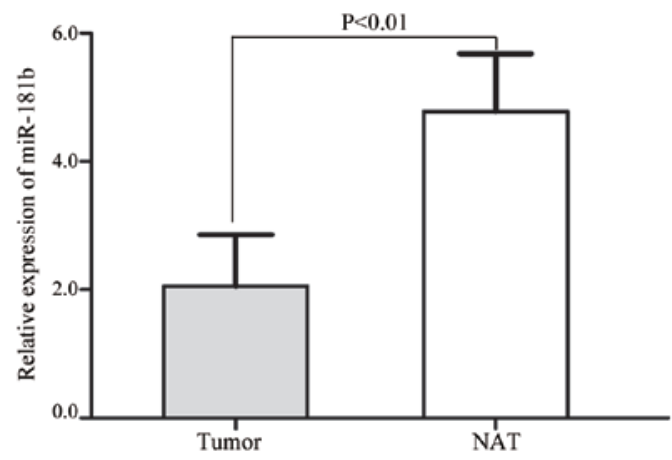

Figure 1. miR-181b is downregulated in NSCLC tissues. The relative expression levels of miR-181b were evaluated by reverse transcription-quantitative polymerase chain reaction in 62 paired NSCLC tissues and NATs. miR, microRNA; NSCLC, non-small cell lung cancer; NAT, normal adjacent tissue.

(StataCorp LP, College Station, Texas, USA). Two-tailed P-values of $<0.05$ were considered to indicate a statistically significant difference.

\section{Results}

miR-181b expression in NSCLC tissues and its association with clinicopathological factors. A total of 62 NSCLC tissues were included in this study. As shown in Fig. 1, miR-181b was significantly downregulated in the NSCLC tissues compared with the NATs $(\mathrm{P}=0.004)$. These results indicated that miR-181b may have a suppressive role in NSCLC.

The study then examined whether the expression level of miR-181b is associated with clinicopathological factors. The statistical analysis showed that miR-181b expression was significantly associated with the tumor-node-metastasis (TNM) classification and metastasis (Table I).

miR-181b inhibits cell migration and invasion in NSCLC H23 and H522 cells. Next, the suppressive role of miR-181b on cell migration and invasion was investigated using the Transwell assay. As shown in Fig. 2, the migration and invasion of the NSCLC H23 ( $\mathrm{P}=0.032$ for migration; $\mathrm{P}=0.280$ for invasion) and $\mathrm{H} 522$ ( $\mathrm{P}=0.020$ for migration; $\mathrm{P}=0.017$ for invasion) cells transfected with miR-181b was markedly decreased compared with the NC. These results indicated that miR-181b decreased the migration and invasion in the NSCLC cells.

HMGB1 is a direct target gene of miR-181b in NSCLC. To identify the target of miR-181b in NSCLC, a public database (TargetScan; http://www.targetscan.org) was used. HMGB1 was predicted to be a target of miR-181b (Fig. 3A). To verify whether miR-181b directly targets HMGB1, western blotting was performed to investigate whether HMGB1 was decreased after transfection with miR-181b in NSCLC H23 and H522 cells. As shown in Fig. 3B, HMGB1 was significantly downregulated in the NSCLC cells following transfection with 

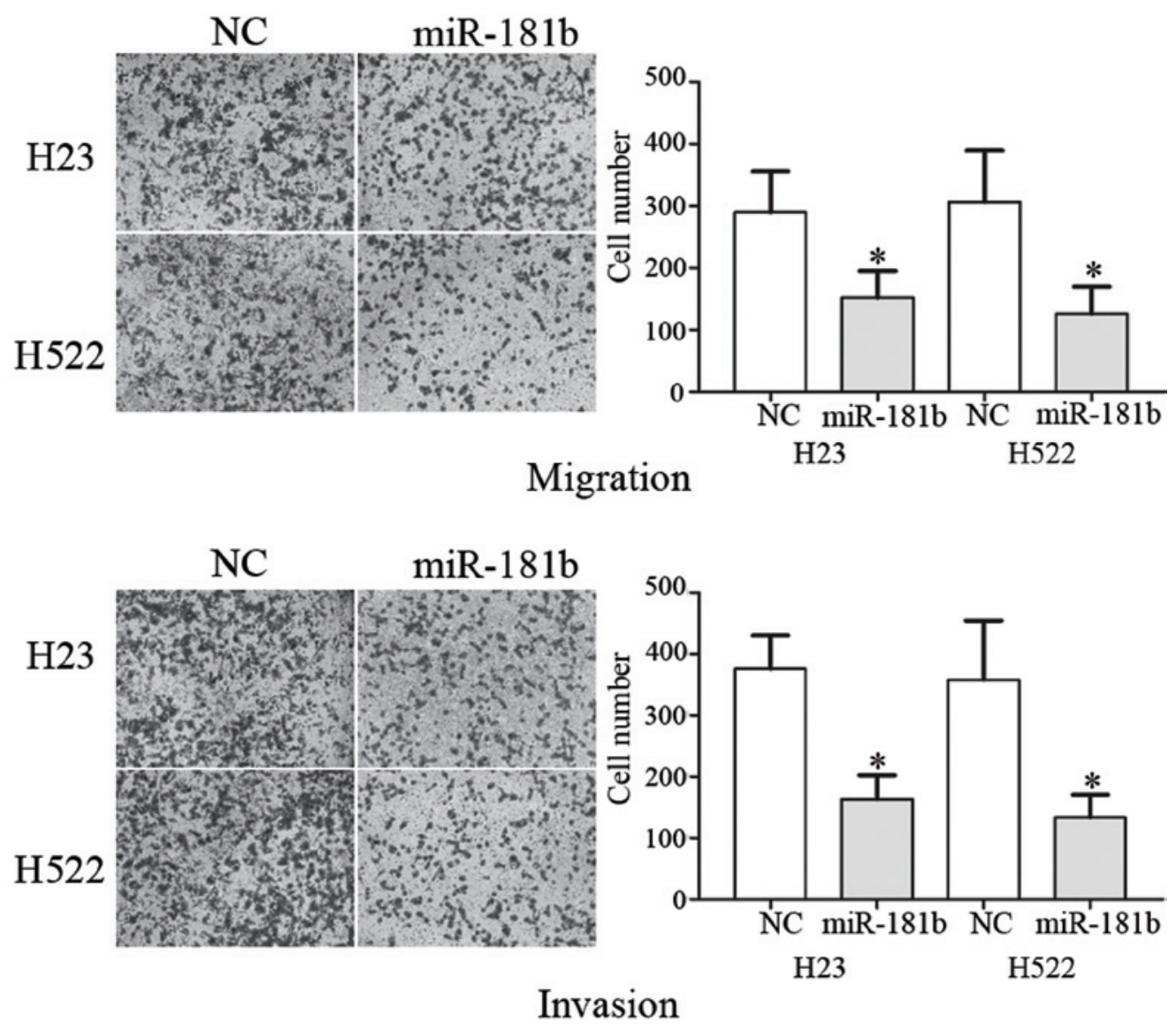

Figure 2. miR-181b inhibits the migration and invasion of NSCLC cells in vitro. The migration and invasion abilities of NSCLC cells transfected with miR-181b mimic and $\mathrm{NC}$ were tested in Transwell chambers with an $8-\mu \mathrm{m}$ pore polycarbonate membrane. miR, microRNA; NSCLC, non-small cell lung cancer; NC, negative control. ${ }^{*} \mathrm{P}<0.05$ compared with respective control.

A

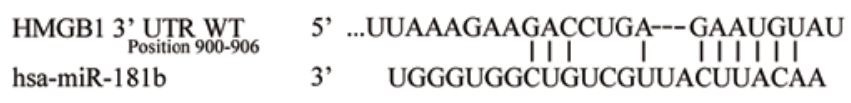
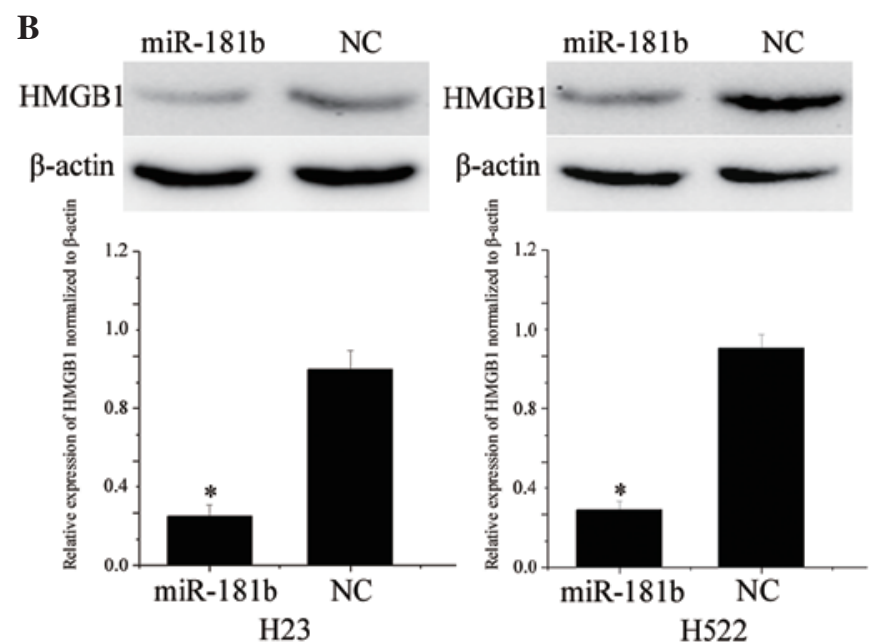
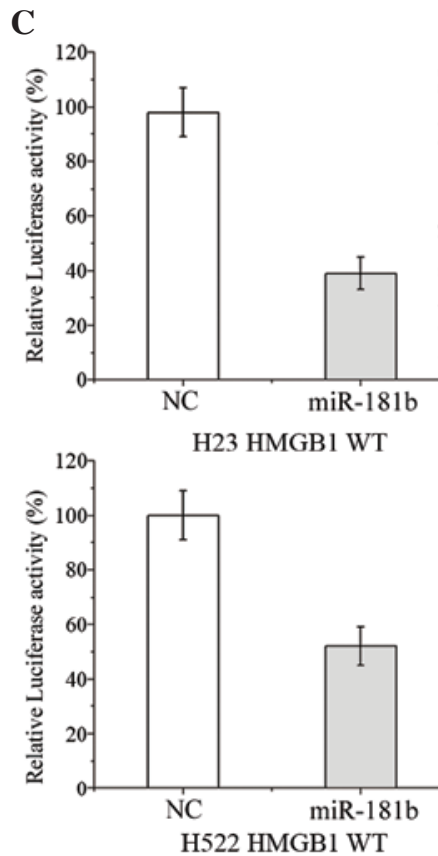

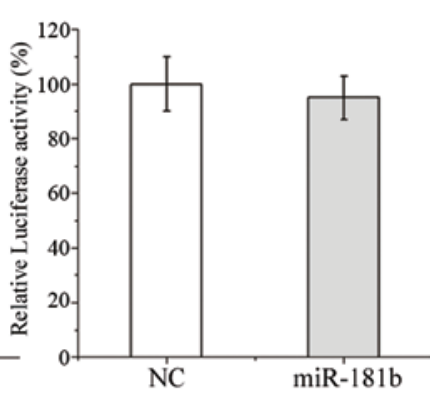

H23 HMGB1 Mut

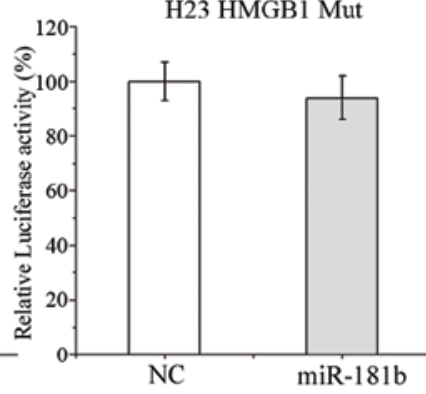

Figure 3.(A)TARGETSCAN assessed that HMGB1 mRNA contains a miR-181b6-nucleotide seed match at position 900-906 of the HMGB1 3'-UTR.(B) Downregulation of HMGB1 after transfection of miR-181b. Western blot analysis was performed to detect the expression levels of HMGB1 protein in miR-181b-transfected and NC-transfected NSCLC cells. $\beta$-actin was used as an internal loading control. (C) Luciferase assay was performed in the NSCLC cells cotransfected with miR-181b mimics and a luciferase reporter containing the WT or Mut 3'-UTR of HMGB1. Luciferase activities were measured at $48 \mathrm{~h}$ post-transfection. Overexpression of miR-181b significantly inhibited the WT but not the Mut luciferase activity of HMGB1 in the NSCLC cells. WT, wild-type; Mut, mutant; NC, negative control; miR, microRNA; HMGB1, high-mobility group box-1; NSCLC, non-small cell lung cancer; UTR, untranslated region. "P<0.05 compared with respective control. 
miR-181b when compared with the $\mathrm{NC}(\mathrm{P}=0.015$ and 0.018 for H23 cells and H522 cells, respectively).

Furthermore, luciferase reporter assays were conducted. Luciferase reporter assays showed that miR-181b inhibited the WT ( $\mathrm{P}=0.026$ for $\mathrm{H} 23$; $\mathrm{P}=0.350$ for $\mathrm{H} 522$ ) but not the MUT luciferase activity of HMGB1 in the NSCLC H23 and H522 cells (Fig. 3C). Overall, HMGB1 was a direct target gene of miR-181b in NSCLC.

\section{Discussion}

Lung cancer is considered to be the leading cause of cancer-related mortality (24). The poor prognosis of patients with early-stage lung cancer is associated with lymph node metastasis and distant metastasis at the time of presentation (25). A previous study revealed that lymph node metastasis indicated high tumor malignancy and a poor prognosis; the patients usually succumbed to the associated complications caused by metastatic loci, but did not succumb to the primary loci-induced symptoms (26). Distant metastasis is a key factor to determine a patient's prognosis, and is one of the most common causes for the failure of treatment for NSCLC (27). The processes inducing and stimulating metastasis are complex and remain unclear. Therefore, an urgent requirement to search for novel sensitive, reliable biomarkers, and novel therapeutic targets and approaches must be sought to block NSCLC metastasis.

miR-181b belongs to the miR-181 family, which comprises four members: miR-181a, miR-181b, miR-181c and miR-181d (20). Previous studies have measured the altered level of miR-181b in multiple tumors and in leukemia/lymphoma (20). The miRNA functions as a tumor suppressor or a tumor promoter in different human malignancies. In one study, miR-181b was found to be downregulated in aggressive B-cell chronic lymphocytic leukemia compared with chronic lymphocytic leukemia (21). In human glioma, the expression of miR-181b was downregulated and the transfection of miR-181b inhibited cell growth, apoptosis and invasion (22). By contrast, Jiang et al verified that miR-181b was significantly upregulated in gastric tumor tissues compared with normal gastric tissues. Kaplan-Meier survival analysis also found that the low expression of miR-181b may be closely associated with better patient overall survival (28). The present study expanded on the data on the expression and function of miR-181b in cancer.

The present results provided evidence that miR-181b was downregulated in NSCLC, which prompted the hypothesis that miR-181b may have a tumor suppressive role in NSCLC carcinoma development and progression. Through the overexpression of miR181b in two NSCLC cell lines, the present study notably showed that miR-181b reduced cell migration and invasion, also suggesting a tumor suppressive role of miR-181b in metastasis. Thus, these results may have clinical implications in the future.

The identification of miR-181b target genes is critical for understanding the role of the miRNA in tumorigenesis, and it is also important for searching for novel therapeutic targets. Studies have verified that $\mathrm{miR}-181 \mathrm{~b}$ regulates oncogenic expression in human cells, including CYLD (29), NOVA1 (30), MDM2 (16), HMGB1 (31), MCL-1 (31), LATS2 (32) and
Bcl-2 (33) cells. Therefore, upregulating miR-181b or providing analogous pharmaceutical compounds exogenously should be effective cancer therapies resulting from the upregulation of these oncogenic transcripts. In the present study, the results showed that miR-181b inhibited cell migration and invasion by directly targeting HGMB1. This finding suggested that miR-181b could be used for the development of novel therapeutic treatments for NSCLC.

HMGB1, located on human chromosome 13q12, is a member of the high mobility group protein super-family (34), and has been reported to be involved in multiple biological processes, including cancer progression, angiogenesis, invasion and metastatic development (35). The upregulation of HMGB1 is found in almost all types of tumors (36). HMGB1 has also been found to be downregulated in NSCLC at the mRNA and protein levels, and is correlated with cancer progression, cell proliferation and metastasis $(36,37)$. The findings suggest that HMGB1 possesses an oncogenic role and is a potential novel therapeutic target. In the present study, it was found that miR-181b controls HMGB1 expression to regulate NSCLC cell migration and invasion. As a result, further investigation is required into its predictive value for the early detection of tumor recurrence and in target therapy drugs to block the invasive nature of NSCLC.

In conclusion, the present study showed that miR-181b was downregulated in NSCLC, and associated with the TNM classification and metastasis. It was also verified that miR-181b inhibits NSCLC cell metastasis by the downregulation of HMGB1. Future studies are required to address whether the predictive and therapeutic potential of miR-181b may be fully realized in cancer treatment. If so, the use of miR-181b may be of future benefit for the treatment of patients with NSCLC.

\section{References}

1. He D, Wang J, Zhang C, Shan B, Deng X, Li B, Zhou Y, Chen W, Hong J, Gao Y, et al: Down-regulation of miR-675-5p contributes to tumor progression and development by targeting pro-tumorigenic GPR55 in non-small cell lung cancer. Mol Cancer 14: 73, 2015.

2. DeSantis CE, Lin CC, Mariotto AB, Siegel RL, Stein KD, Kramer JL, Alteri R, Robbins AS and Jemal A: Cancer treatment and survivorship statistics, 2014. CA Cancer J Clin 64: 252-271, 2014.

3. Yang Y, Liu L, Zhang Y, Guan H, Wu J, Zhu X, Yuan J and Li M: MiR-503 targets PI3K p85 and IKK- $\beta$ and suppresses progression of non-small cell lung cancer. Int J Cancer 135: 1531-1542, 2014.

4. Zhang B, Liu T, Wu T, Wang Z, Rao Z and Gao J: microRNA-137 functions as a tumor suppressor in human non-small cell lung cancer by targeting SLC22A18. Int J Biol Macromol 74: 111-118, 2015.

5. Crinò L, Weder W, van Meerbeeck J and Felip E; ESMO Guidelines Working Group: Early stage and locally advanced (non-metastatic) non-small-cell lung cancer: ESMO clinical practice guidelines for diagnosis, treatment and follow-up. Ann Oncol 21 (Suppl 5): v103-v115, 2010.

6. Gupta GP and Massagué J: Cancer metastasis: Building a framework. Cell 127: 679-695, 2006.

7. Yu X, Wei F, Yu J, Zhao H, Jia L, Ye Y, Du R, Ren X and Li H: Matrix metalloproteinase 13: A potential intermediate between low expression of microRNA-125b and increasing metastatic potential of non-small cell lung cancer. Cancer Genet 208: 76-84, 2015.

8. Zhu X, Gao G, Chu K, Yang X, Ren S, Li Y, Wu H, Huang Y and Zhou C: Inhibition of RAC1-GEF DOCK3 by miR-512-3p contributes to suppression of metastasis in non-small cell lung cancer. Int J Biochem Cell Biol 61: 103-114, 2015. 
9. Iorio MV and Croce CM: MicroRNA dysregulation in cancer: Diagnostics, monitoring and therapeutics. A comprehensive review. EMBO Mol Med 4: 143-159, 2012.

10. Engels BM and Hutvagner G: Principles and effects of microRNA-mediated post-transcriptional gene regulation. Oncogene 25: 6163-6169, 2006.

11. van Rooij E, Purcell AL and Levin AA: Developing microRNA therapeutics. Circ Res 110: 496-507, 2012.

12. Chen C, Zhao Z, Liu Y and Mu D: microRNA-99a is downregulated and promotes proliferation, migration and invasion in non-small cell lung cancer A549 and H1299 cells. Oncol Lett 9: 1128-1134, 2015.

13. Ambros V: The functions of animal microRNAs. Nature 431: 350-355, 2004

14. Bartel DP: MicroRNAs: Genomics, biogenesis, mechanism, and function. Cell 116: 281-297, 2004.

15. Broderick JA and Zamore PD: MicroRNA therapeutics. Gene Ther 18: 1104-1110, 2011.

16. Sun YC, Wang J, Guo CC, Sai K, Wang J, Chen FR, Yang QY, Chen YS, Wang J, To TS, et al: MiR-181b sensitizes glioma cells to teniposide by targeting MDM2. BMC Cancer 14: 611, 2014.

17. Ventura A and Jacks T: MicroRNAs and cancer: Short RNAs go a long way. Cell 136: 586-591, 2009.

18. Yang T, Thakur A, Chen T, Yang L, Lei G, Liang Y, Zhang S, Ren $\mathrm{H}$ and Chen M: MicroRNA-15a induces cell apoptosis and inhibits metastasis by targeting BCL2L2 in non-small cell lung cancer. Tumour Biol 36: 4357-4365, 2015.

19. Lewis BP, Shih IH, Jones-Rhoades MW, Bartel DP and Burge CB: Prediction of mammalian microRNA targets. Cell 115: 787-798, 2003.

20. Yang J, Liu H, Wang $H$ and Sun Y: Down-regulation of microRNA-181b is a potential prognostic marker of non-small cell lung cancer. Pathol Res Pract 209: 490-494, 2013.

21. Visone R, Veronese A, Balatti V and Croce CM: MiR-181b: New perspective to evaluate disease progression in chronic lymphocytic leukemia. Oncotarget 3: 195-202, 2012.

22. Shi ZM, Wang XF, Qian X, Tao T, Wang L, Chen QD, Wang XR, Cao L, Wang YY, Zhang JX, et al: MiRNA-181b suppresses IGF-1R and functions as a tumor suppressor gene in gliomas. RNA 19: 552-560, 2013.

23. Livak KJ and Schmittgen TD: Analysis of relative gene expression data using real-time quantitative PCR and the 2(-Delta Delta C(T)) Method. Methods 25: 402-408, 2001.

24. Wang RJ, Zheng YH, Wang P and Zhang JZ: Serum miR-125a-5p, miR-145 and miR-146a as diagnostic biomarkers in non-small cell lung cancer. Int J Clin Exp Pathol 8: 765-771, 2015.
25. Reungwetwattana T, Weroha SJ and Molina JR: Oncogenic pathways, molecularly targeted therapies, and highlighted clinical trials in non-small-cell lung cancer (NSCLC). Clin Lung Cancer 13: 252-266, 2012.

26. Mountain CF and Dresler CM: Regional lymph node classification for lung cancer staging. Chest 111: 1718-1723, 1997.

27. Wang R, Chen XF and Shu YQ: Prediction of non-small cell lung cancer metastasis-associated microRNAs using bioinformatics. Am J Cancer Res 5: 32-51, 2014.

28. Jiang J, Zheng X, Xu X, Zhou Q, Yan H, Zhang X, Lu B, Wu C and $\mathrm{Ju} \mathrm{J}$ : Prognostic significance of miR-181b and miR-21 in gastric cancer patients treated with S-1/Oxaliplatin or Doxifluridine/Oxaliplatin. PLoS One 6: e23271, 2011.

29. Li D, Jian W, Wei C, Song H, Gu Y, Luo Y and Fang L: Down-regulation of miR-181b promotes apoptosis by targeting CYLD in thyroid papillary cancer. Int J Clin Exp Pathol 7: 7672-7680, 2014.

30. Zhi F, Wang Q, Deng D, Shao N, Wang R, Xue L, Wang S, Xia X and Yang Y: MiR-181b-5p downregulates NOVA1 to suppress proliferation, migration and invasion and promote apoptosis in astrocytoma. PLoS One 9: e109124, 2014.

31. Lu F, Zhang J, Ji M, Li P, Du Y, Wang H, Zang S, Ma D, Sun X and Ji C: miR-181b increases drug sensitivity in acute myeloid leukemia via targeting HMGB1 and Mcl-1. Int J Oncol 45: 383-392, 2014.

32. Xia Y and Gao Y: MicroRNA-181b promotes ovarian cancer cell growth and invasion by targeting LATS 2 . Biochem Biophys Res Commun 447: 446-451, 2014.

33. Cai B, An Y, Lv N, Chen J, Tu M, Sun J, Wu P, Wei J, Jiang K and Miao Y: miRNA-181b increases the sensitivity of pancreatic ductal adenocarcinoma cells to gemcitabine in vitro and in nude mice by targeting BCL-2. Oncol Rep 29: 1769-1776, 2013.

34. Ferrari S, Finelli P, Rocchi M and Bianchi ME: The active gene that encodes human high mobility group 1 protein (HMG1) contains introns and maps to chromosome 13. Genomics 35: 367-371, 1996

35. Yao S, Zhao T and Jin H: Expression of MicroRNA-325-3p and its potential functions by targeting HMGB1 in non-small cell lung cancer. Biomed Pharmacother 70: 72-79, 2015.

36. Shen X, Hong L, Sun H, Shi M and Song Y: The expression of high-mobility group protein box 1 correlates with the progression of non-small cell lung cancer. Oncol Rep 22: 535-539, 2009.

37. Sun KK, Ji C, Li X, Zhang L, Deng J, Zhong N and Wu XY: Overexpression of high mobility group protein $\mathrm{B} 1$ correlates with the proliferation and metastasis of lung adenocarcinoma cells. Mol Med Rep 7: 1678-1682, 2013. 